\title{
REGISTRO DE UN EVENTO: \\ EL VI CONGRESO NACIONAL DE PSICOLOGIA
}

Marco Vargas ${ }^{1}$

Entre el 26 y 29 de Octubre de 1993, organizado por el Colegio de Psicólogos del Perú, se llevó a cabo el VI Congreso Nacional de Psicología, teniendo como ambiente físico las instalaciones del Centro Cívico de Lima.

A decir del Comité Organizador, objetivos del evento fueron: (1) Analizar el desarrollo histórico de la psicología en el Perú y Latinoamérica; (2) Examinar la situación actual de la psicología como ciencia y profesión y (3) Evaluar los retos de nuestra ciencia en el momento actual y plantear alternativas para su desarrollo en perspectiva al siglo XXI.

Las ponencias fueron presentadas en seis salas a través de ciento cuarentiseis sesiones (algunas no se llevaron a cabo por inasistencia de los expositores). Una idea cabal del evento nos lo da el siguiente cuadro:

\section{CIFRAS DEL VI CONGRESO NACIONAL DE PSICOLOGIA* Lima, 1993}

\begin{tabular}{lcccc}
\hline Modalidad de Trabajo & $\mathbf{N}^{\circ}$ de Sesiones & $(\%)$ & $\mathbf{N}^{\circ}$ de Ponencias & $(\%)$ \\
\hline Conferencias & 27 & 18.5 & 27 & 9.9 \\
Mesas Institucionales & 27 & 18.5 & 46 & 16.8 \\
Conferencia por invitación & 22 & 15 & 22 & 8 \\
Comunicaciones libres & 22 & 15 & 90 & 32.9 \\
Talleres & 22 & 15 & 22 & 8 \\
Mesas redondas & 13 & 9 & 56 & 20.4 \\
Simposias & 5 & 3.4 & 5 & 1.8 \\
Relatos Oficiales & 4 & 2.7 & 4 & 1.4 \\
Presentación de literatura & 2 & 1.4 & 2 & 0.7 \\
de autores nacionales & 2 & 1.4 & - & - \\
Inauguración - Clausura & 146 & 99.9 & 274 & 99.9 \\
Total & & & & \\
\hline
\end{tabular}

* Fuente: Programa Oficial del VI Congreso Nacional de Psicología; Lima, 26-29 de Octubre de 1993.

1. Bachiller en Psicología de la Universidad Ricardo Palma. 
Las conferencias (ordinarias y por invitación), fueron las modalidades más resaltantes; en conjunto hubieron cuarentinueve sesiones para igual número de ponencias -como modalidad de trabajo representaron un tercio de ellas (33.6\%) y como ponencias el $17.9 \%$. Entre las conferencias por invitación, hubieron aquellas cuyas temáticas fueron: "Educación Especial" (Liliana Mayo); "Psicolingüística" (Raúl González); "Psicología Organizacional" (Alvaro Gonzales); "Nuevas Orientaciones en Psicología del Desarrollo" (Cecilia Thorne); "Psicología Social" (Carmen Pimentel); "Investigación en Psicología" (Reynaldo Alarcón); "Drogas" (Delicia Ferrando); "Psicología Comunitaria" (Carlos Marchena); etc. Las mesas institucionales tuvieron espacio en veintisiete sesiones -en orden de preferencias ocupó el segundo lugar como modalidad de trabajo asumido- habiéndose sustentado en ellas cuarentiseis ponencias. El mayor número de instituciones que acreditó su asistencia al evento recayó en las universidades; estuvieron presentes todas aquellas que tienen una Facultad de la especialidad de psicología y que se ubican en la capital peruana (UNMSM; UNFV; PUCP; UPCH; UL; UNIFE; UIGV Y USMP), así como dos del interior (San Agustín de Arequipa y Hermilio Valdizán de Huánuco). El segundo grupo de instituciones que ocupó una mesa, estuvo a cargo de ocho organizaciones (entre ellas CEDRO, INBIF, IFASIL, INTERCOMSEX, etc.), siguiendo luego instituciones de salud (INSM "H. Delgado - H. Noguchi"; HC de la Policía Nacional del Perú y varias clínicas de salud mental) y asociaciones de psicólogos. En general, las mesas institucionales estuvieron dedicadas a la aplicación de la psicología en diversas áreas y al estado actual en que se encuentra ésta. Las comunicaciones libres tuvieron lugar en veintidos sesiones, sustentándose en ellas noventa ponencias (32.9\% del total), con un espectro temático muy variado en cada una de las sesiones en que fueron incluidas. Los talleres, al igual que las comunicaciones libres, alcanzaron veintidos sesiones para idéntico número de ponencias, abundando aquellas cuyas temáticas se referían a técnicas terapéuticas (ocho) y, en menor grado de incidencia, aquellas que se referían a problemas de aprendizaje (tres). Las mesas redondas ocuparon trece sesiones - casi la décima parte entre las modalidades de trabajo optadas- sustentándose cincuentiseis ponencias $(20.4 \%$ del total). Aquí, las temáticas abordadas fueron claras y precisas; cuatro sesiones estuvieron dedicadas a la farmacodependencia (dos al tema de la comunidades terapéuticas, una a modelos de tratamiento y otra dedicada a las actividades de prevención); otras cuatro, centraron su atención en el SIDA (dós al tema de los aspectos psicológicos del paciente portador del VIH, uno dedicada a la situación del síndrome en el Perú y el mundo y, otro, a los hallazgos psicopatologicos e intervención terapéutica), en un idéntico número de sesiones se abordó la problemática de la violencia (infantil, familiar, social, -niños de la calle- y contra la mujer), finalmente, una sesión de mesa redonda estuvo dedicada a la psicología deportiva. Las simposias se desarrollaron en cinco sesiones (los temas fueron los siguientes: "Retos y alternativas de los proyectos educativos en problemas de 
aprendizaje"; "La Revista de Psicología de la Pontificia Universidad Católica del Perú"; "Epistemología y Psicología"; "Avances en Neuro-Psicología" y "Aproximaciones terapéuticas"). Para finalizar, el congreso dio cabida en cuatro sesiones a un igual número de relatos oficiales (cuyos contenidos apuntaban a cristalizar los objetivos del evento), los mismos que se desarrollaron a primera hora durante cada uno de los días que duró el congreso.

En éste recuento, se hace notoria -de manera transparente- una de las funciones de todo congreso: ser un medio o canal de comunicación de toda ciencia, potenciando el crecimiento de la necesidad de encontrarse, agruparse y colaborar. Además, es evidente también, la utilidad de los congresos al permitir éstos hacer un balance formal de la especialidad o disciplina que las convoca -siendo ésto válido para cada ocasión que suceda - toda vez que en ellos se refleja lo más reciente, lo "ultimito" de los resultados e intereses de investigación de todos aquellos quienes acuden a cada evento a presentar sus ponencias.

Es evidente que la evaluación de cada congreso o el conjunto de éstos en un intervalo de tiempo, es una forma de evaluar el desarrollo y madurez (también el estancamiento e involución) de una ciencia. En un contexto amplio, por ejemplo, los Congresos Internacionales de Psicología -que datan desde 1889han merecido atención para su estudio ${ }^{1}$, igualmente los Congresos Interamericanos -que se iniciaran en 1953- celebrados en nuestro medio en dos ocasiones (1966 y 1979), han sido evaluados, analizándose las características de la psicología en el Perú a través de las ponencias de nuestros connacionales ${ }^{2}$. Sin embargo, los Congresos Nacionales de Psicología (ya celebrados en seis ocasiones), no han sido evaluados. Sólo se ha registrado el primer evento a través de una memoria ${ }^{3}$, que es muy probable que siga siendo la única. Es obvio que desde la ya lejana segunda década del setenta, estamos perdiendo (¿seguiremos perdiendo?) el rastro del desarrollo, evolución y tendencias que los psicólogos peruanos han ido "edificando" cada vez que podían mostrar sus "obras" en la construcción de la psicología en el Perú. Esto seguirá sucediendo toda vez que no registremos el presente individual y colectivo. En el futuro lo pretérito será difícil de recordar por falta de "memoria".

1. Montoro, L; Carpintero, H. y Quintanilla, I. (1982) Comunicación científica en los Congresos Internacionales de Psicología (I-XVI). Revista de Historia de la Psicología. 1; 43-62.

2. León, R. y Salas, R. (1986) Trece años después: Una aproximación a la psicología peruana en 1966 y en 1979. Revista de la Universidad Ricardo Palma, 8-9; 67-76.

3. Alarcón, R.; Infante, J.; Ponce, C. y Bibolíni, A.

[Edits.].(1975) La investigación psicológica en el Perú. Memorias del primer Congreso Peruano de Psicología. Lima: Sociedad Peruana de Psicología. 
Lo más "fresco" de las "huellas" de la psicología que se desarrolla en nuestro medio -y que puede ser comparado con lo observado en el III Congreso Nacional de Psicología realizado hace una década y que fuera "registrado" en ésta misma revista- ${ }^{4}$, puede ser visualizado en el cuadro.

El análisis del cuadro nos permite identificar las ponencias sustentadas por áreas. Notorio es el predominio de la psicología social como área de interés -tanto temático como de aplicación- entre los psicólogos de nuestro medio. Sin embargo, la psicología entendida como una ciencia de la salud, es la que se impone; las ponencias en el área clínica sumadas a las del área de la psicoterapia, superan a las del área social $(24.1 \%$ vs $17.7 \%)$. Esto no es novedad, por el contrario, confirma y consolida el tradicional interés que ésta área de la psicología concita entre nosotros desde siempre. Ya en los Congresos Interamericanos de 1966 y 1979, celebrados en Lima (2), así como en los Congresos Nacionales de 1981 y 1983 (4), la psicología clínica ocupó el primer lugar de interés. Habría que señalar -por ser sumamente evidente- que en 1981 el Análisis y Modificación de la Conducta (aplicación terapéutica de la clínica) fue la gran abanderada. Doce años después, su presencia es casi nula a pesar de tener una actividad permanente (existe una Sociedad Peruana de Terapia y Modificación del Comportamiento).

Incuestionablemente, la psicología clínica es la especialidad que cuenta con mayor número de adeptos, esto estaría sugiriendo que entre los interesados en estudiar psicología, lo harían mirando en ella una carrera profesional liberal y por tanto indenpendientes, a la manera de la medicina -la más representativa entre las ciencias de la salud- fenómeno éste casi generalizado en Latinoamérica ${ }^{5}$.

La preferencia -también constante en el tiempo- por la psicología social (aparentemente en primer orden en el último evento congresal), tiene su explicación en la complicada situación de una sociedad como la nuestra, con material tan propicio -no siempre adecuadamente identificado y menos abordado- para su estudio, por ejemplo, este VI Congreso Nacional dedicó atención al tema cotidiano de la violencia en algunas de sus modalidades -decreciente en los últimos tiempos en su expresión a través de la subversión y el terrorismo y, prácticamente no estudiada por los psicólogos entre la población afectada por

4. Salas, R. (1983) Artículo-reseña: El tercer Congreso Nacional de Psicología y la ciencia psicológica en el Perú. Revista de Psicología, PUCP. 1 (2); 171-174.

5. Ardila, R. (1985) Factores Socioculturales en el desarrollo de la psicología: el caso de América Latina. En: Carpintero, H. y Peiró, J.M. [Dirs.] (1985) La psicología en su contexto histórico. Ensayos en honor del Prof. Josef Brozek. Monografía de la Revista de Historia de la Psicología; 41-47. 


\section{Lima-1993}

\begin{tabular}{|c|c|c|c|c|c|}
\hline \multirow{2}{*}{\multicolumn{2}{|c|}{$\begin{array}{l}\text { Ponencias por áreas a las que pertenecen } \\
\text { por su orientación y el tema que tratan }\end{array}$}} & \multicolumn{4}{|c|}{ PORCENTA JES } \\
\hline & & 0 & 10 & 15 & 20 \\
\hline Psic. Social & 47 & E. & & & $17.7 \%$ \\
\hline Psic. Clínica y de la Salud & 34 & Fing & ? & & \\
\hline Psicoterapia & 30 & ming & (2) & & \\
\hline Asuntos Profesionales & 21 & 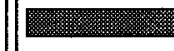 & $7.9 \%$ & & \\
\hline Psic. Indust. y Organizac. & 15 & Fin: & & & \\
\hline Psic. General & 15 & 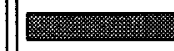 & & & \\
\hline Psic. Educativa & 14 & F & & & \\
\hline Psicometría & 11 & 4. & & & \\
\hline Psic. del Aprendizaje & 8 & 3.0 & & & \\
\hline Comportamiento Sexual & 7 & $2.6 \%$ & & & \\
\hline Psicoformacología & 7 & $2.6 \%$ & & & \\
\hline Psic. de la Comunic. Soc. & 6 & $2.2 \%$ & & & \\
\hline Psic. Deportiva & 6 & $2.2 \%$ & & & \\
\hline Historia y Sistemas & 5 & $1.9 \%$ & & & \\
\hline Personalidad & 5 & $1.9 \%$ & & & \\
\hline Psic. Evolutiva & 4 & $1.5 \%$ & & & \\
\hline Psic. Gerontológico & 4 & $1.5 \%$ & & & \\
\hline Neuropsicología & 4 & $1.5 \%$ & & & \\
\hline Psic. Fisiológica & 3 & $1.1 \%$ & & & \\
\hline Metodología & 3 & $1.1 \%$ & & & \\
\hline Psic. Humanística & 3 & $1.1 \%$ & & & \\
\hline Psic. Cognitiva & 3 & $1.1 \%$ & & & \\
\hline Psic. Criminológica & 2 & $0.8 \%$ & & & \\
\hline Motivación y Emoción & 2 & $0.8 \%$ & & & \\
\hline Pensamiento $\dot{y}$ Lenguaje & 2 & $0.8 \%$ & & & \\
\hline Psicolingüística & 2 & $0.8 \%$ & & & \\
\hline Biorretroalimentación & 1 & $0.4 \%$ & & & \\
\hline Psic. Transcultural & 1 & $0.4 \%$ & & & \\
\hline Psicoanálisis & 1 & $0.4 \%$ & & & \\
\hline Total de Ponencias & 266 & $100.0 \%$ & & & \\
\hline
\end{tabular}

* Datos elaborados en base al Programa Oficial del VI Congreso Nacional de Psicología Lima; 26-29 de octubre de 1993. 
ella, habiendo, por supuesto, algunas excepciones- ${ }^{6}$. Durante la presente década, problemas psicosociales como el maltrato infantil, familiar, hacia la mujer, los factores de dicho orden entre pacientes con SIDA y con problemas de adicción a drogas (el primero, tema novísimo y de creciente interés a partir de mediados de la década del ochenta, cuando hace su aparición en nuestro país y el segundo, tema "tradicional" desde que la problemática del consumo de sustancias psicoactivas se hace evidente en la población peruana desde la década del sesenta), son los temas que interesan a la psicología en el Perú. Sin embargo, lenta y manifiestamente en los últimos años, quizás como una expresión de "compromiso social", la psicología comunitaria -término acuñado a mediados de la década del sesenta- ${ }^{7}$, para algunos una nueva forma de psicología aplicada no exclusivamente social por sus vínculos con los aspectos clínicos, viene ganando un creciente espacio entre los intereses de los psicólogos peruanos; casi un $20 \%$ de ponencias en el área social ( $\mathrm{N}=47$ ), corresponden a ella, además de evidenciarse también, un asomo de interés por la psicología de la comunicación social.

El tema de los asuntos profesionales ocupó el cuarto lugar de interés, aunque en esencia, una suma de las ponencias en el área educativa y la psicología del aprendizaje (que mayormente se refirió a los problemas de aprendizaje entre escolares), superó ligeramente a ésta $(8.3 \%$ vs $7.9 \%)$.

Es evidente que luego de la psicología clínica y social, interesa a los psicólogos en el Perú, los temas concernientes a la psicología educacional, interés que tiene un largo pasado y debe sus raíces históricas a las semillas que sembró Walter Blumenfeld (1882-1967), quién durante la primera mitad de la década del cuarenta colaboró y participó activamente en la formación y labores del Instituto Psicopedagógico Nacional, dirigiendo incluso, a partir de 1955, el Instituto de Psicopedagogía de la Facultad de Educación de la Universidad de San Marcos y llegando a publicar un libro -que alcanzó cinco ediciones- dedicado precisamente a la psicología del aprendizaje. La década del setenta, gracias a ese pasado inmediato, pudo - a través del INIDE- ofrecer a los psicólogos peruanos, políticas de investigación más definidas en el campo de la psicología educacional, al punto que ésta línea temática -quizás la que mejor estátus científico ha alcanzado- ha

6. León, R. y Zambrano, A. (1990) Efectos psicosociales de los cortes de luz y agua en un grupo de mujeres de una zona deprivada de Lima Metropolitana. Socialismo y Participación, 52; 1-18.

7. Montero, M. (1990) Perspectivas de la psicología comunitaria en América Latina. Psicología Actual, 3 (7); 17-29. 
merecido estudios dedicados a su análisis, diagnóstico y perspectivas en nuestro medio ${ }^{8} \mathrm{y}^{9}$.

Los asuntos profesionales, tema que siempre ha llamado la atención a nuestros psicólogos, parece haber alcanzado durante el último congreso, la mayor cúspide de interés en los últimos años. El hecho de que el evento que comentamos haya tenido como uno de sus objetivos evaluar la situación actual, así como los retos de la psicología como ciencia y profesión en el Perú, explicarían su alta incidencia en la gradiente de intereses de los psicólogos peruanos. Cabe preguntarnos: ¿el auge de éste interés, es el incremento de una creciente búsqueda de identidad de la psicología en el Perú?, ¿Se estarán planteando realmente, en nuestro contexto, nuestros problemas?, ¿los estaremos reconociendo?. Indudablemente, la psicología es una ciencia joven y más joven lo es aún en el Perú. Quizás estaremos madurando (o quizás nos planteamos el verbalismo fácil de la "importancia de la psicología"), en todo caso, nuevas y próximas ponencias, así como resultados de investigaciones y todo cuanto sea fruto de los actuales afanes de los psicólogos peruanos, develaran la razón de tan creciente interés.

La psicología industrial y organizacional casi desapercibida en los sesenta, se hace perceptible entre nosotros a mediados de la década del setenta ${ }^{10}$. A partir de ahí, el interés de los psicólogos peruanos en dicha área ha ido creciendo y su registro en revistas y congresos ha sido manifiesto, no en las proporciones de la psicología educacional, social y menos aún clínica, pero casi de un modo permanente ha tenido su presencia . (esta vez en una proporción porcentual ligeramente mayor a lo evidenciado en otros congresos nacionales como el segundo y el tercero). Es probable que en una economía de libre mercado -como es la que se viene instaurado en el Perú de los noventa- la psicología aplicada al área laboral, habrá de cobrar algún impulso.

La tendencia psicometrista de la década del setenta -advertida en la mitad de las comunicaciones libres que fueran presentadas al 1 Congreso Nacional de Psicología-(2), sigue expresando su "fuerza" pues, se impone a otras orientaciones temáticas, cierto que en proporciones porcentuales mínimas, pero mayores al fin. Temáticas como las referidas al comportamiento sexual, la psicofarmacología, la psicología de la comunicación social y la psicología deportiva, alcanzan una posición intermedia entre los intereses manifiestos de los psicólogos peruanos

8. Meza, A. (1988) Psicología Educacional en el Perú. Psicología y Sociedad, 1 (1); 17-94.

9. Lazarte, C. (1991) Análisis, diagnóstico y perspectivas de la investigación psicoeducacional en el Perú. Revista de Psicología, PUCP. 9 (1); 37-87.

10. León, F. (1982) La investigación psicólogica del trabajo y las organizaciones en el Perú: 19561981. Socialismo y Participación, 19; 61-71. 
de hoy, sobre todo las dos primeras, que quizás siga creciendo a medida que vaya -como parece ser- agudizándose la problemática del SIDA y de las drogas en el Perú.

La presencia de un área temática como la historia de la psicología, situada a "medida tabla" (con una presencia porcentual cercana al 2\%) entre las ponencias presentadas, se debe más que a un auténtico interés por ella entre los profesionales de la especialidad de nuestro medio, a los relatos oficiales del evento, los mismos que se ocuparon de la historia de las ideas psicológicas en Latinoamérica y el Perú, además de esbozar un panorama actual de ella y avizorar algunos retos y alternativas que le esperan en el futuro.

Entre las áreas de interés con una menor preferencia, ubicamos a la psicología evolutiva. Sin embargo, creemos que ese no es auténticamente el lugar que le corresponde. Es muy probable que si asignamos ponencias por áreas temáticas conociendo -además del título- las características muestrales y los fines de las investigaciones que fueran expuestas en el congreso (datos que pueden ser identificados en los resúmenes que contiene una memoria) descubriríamos que en varias, sino en muchas de ellas, se presta atención a los problemas de la niñez y adolesencia. Ya a mediados de la década del ochenta era identificable la preminencia de trabajos nacionales ${ }^{11}$, miembros también, de una población mayoritariamente joven como lo demuestran los indicadores demográficos peruanos. Esa "juventud", también ayudaría a comprender la poca presencia de ponencias en el área de la psicología gerontológica.

La neuropsicología, psicofisiología y biorretroalimentación "pintan" tenuemente en el amplio espectro de áreas que tienen la psicología (de las cuales sólo 29 son consignados en el cuadro que hemos elaborado). En medida alguna, ocupan lugares esperados o "normales" para el grado de desarrollo que viene alcanzando la psicología en el Perú, donde prácticamente no se cuenta con laboratorios, equipos idóneos ni psicólogos con una formación claramente experimental (la ausencia de ponencias en el área de la psicología experimental puede, buenamente, explicarse por ello). En todo caso, la investigación aplicada en psicología es la que a lo psicólogos peruanos viene interesando sostenidamente desde hace tres décadas. En cambio, en dicho intervalo de tiempo, al parecer, el psicoanálisis viene perdiendo la fuerza que demostrará al interesar a los psicólogos de los sesenta y setenta.

Aquí, debemos señalar que este hecho no significa que el psicoanálisis no tenga presencia en nuestro país. Honorio Delgado (1892-1969), difundió la

11 León, F. [Edit.] (1986) Psicología y realidad peruana. El aporte objetivo Lima: Mosca Azul. 
creación freudiana entre la segunda y tercera década de la presente centuria, otro tanto lo hizo Carlos Alberto Seguín desde los años cuarenta y, desde fines de los sesenta, el psicoanálisis en el Perú ingresa a una etapa de mayor auge académico y profesional (gracias a la labor de analistas como Saúl Peña, Carlos Crisanto y Max Hernández), alcanzando cierto nivel de organización en 1980, al fundarse la Sociedad Peruana de Psicoanálisis . Actualmente, el movimiento psicoanalista en nuestro medio tiene una "vida" activa, expresada en publicaciones y eventos congresales propios de dicha área.

En general, diremos que una comparación entre el III Congreso Nacional de Psicología (4) y la sexta versión de dicho evento que aquí comentamos, evidencia un notorio aumento de la productividad de los psicólogos nacionales a través del número de sesiones dedicadas a las diversas modalidades de trabajo congresal. Así, en 1983 hubieron cincuenticuatro sesiones en tres días de duración que tuvo el evento (16-18 de Dic.) y diez años después, el número de éstas ascendió a ciento cuarentiseis (casi tres veces más), en cuatro días de sesiones; las mesas institucionales variaron de 9 a 27; las conferencias por invitación y las comunicaciones libres variaron de 13 a 22 y las mesas redondas de 9 a 13 . El número de simposias se ha mantenido en cinco y a diferencia del evento de 1983, que incluyó cinco sesiones de conversatorios, éste último no lo hizo, añadiendo sí, modalidades como las conferencias, talleres y relatos oficiales. Probablemente los congresos nacionales vienen constituyéndose en los medios y canales de comunicación académica y profesional más preferidos por los psicólogos peruanos, más aún, si la actividad editorial en nuestro país es una "aventura riesgosa" en el frente financiero (hecho que estaría explicando la ausencia de ediciones de cada una de las memorias correspondientes a los congresos nacionales), aunque al parecer, la década de los noventa es más auspiciosa para los afanes publicistas pues, hay que saludar que a la continuidad de la Revista de Psicología de la Pontificia Universidad Católica del Perú -que ha merecido un estudio y elaboración de una guía hemerográfica (lo primero por ser una muestra de la producción bibliográfica de la psicología en el Perú y lo segundo, para ofrecer una mayor cantidad de "ingresos" a la Revista y sistematizar sus contenidos) $-{ }^{12}$, se suman otras que pretenden también mantener vigencia (v.g. Revista de Psicología de la Universidad Ricardo Palma; Psicología Actual; Psicoactiva) o resultan ser novedosas (como la Revista de Problemas de Aprendizaje, editada por el Colegio Palestra y Más Luz-Revista de Psicología y Pedagogía- editada por el Colegio Domingo F. Sarmiento), constituyéndose

12 Meza, A.; Quintana, A. y Lostaunau, G. (1993) La producción psicológica en el Perú. Una mirada a través de la Revista de Psicología de la Pontificia Universidad Católica del Perú. Revista de Psicología, PUCP. Volúmen extraordinario. 
-congresos y publicaciones- como las vías en las que se registran los "pasos" que la psicología ( $y$ cualquier otra ciencia) va dando en su desarrollo, ya sea en nuestro medio y en cualquier otra latitud. 